\title{
Boundary element method for optical force calibration in microfluidic dual- beam optical trap
}

Mehmet E. Solmaz

Barbaros Çetin

Besim Baranoğlu

Murat Serhathoğlu

Necmi Biyikli 


\title{
Boundary Element Method for Optical Force Calibration in Microfluidic Dual-Beam Optical Trap
}

\author{
Mehmet E. Solmaz ${ }^{a, b}$, Barbaros Çetin $^{c}$, Besim Baranoğlu $^{d}$ \\ Murat Serhatlıŏ lu ${ }^{b}$, Necmi Bıyıklı \\ ${ }^{a}$ Dept. Electrical and Electronics Eng., İzmir Katip Çelebi University, İzmir 35620, Turkey; \\ ${ }^{b}$ National Nanotechnology Research Center, I.D. Bilkent University, Ankara 06800, Turkey; \\ ${ }^{c}$ Microfluidics \& Lab-on-a-Chip Research Group, Mechanical Engineering Department \\ I.D. Bilkent University, Ankara 06800, Turkey; \\ ${ }^{d}$ Department of Manufacturing Engineering, Atılım University, Ankara 06836 Turkey
}

\begin{abstract}
The potential use of optical forces in microfluidic environment enables highly selective bio-particle manipulation. Manipulation could be accomplished via trapping or pushing a particle due to optical field. Empirical determination of optical force is often needed to ensure efficient operation of manipulation. The external force applied to a trapped particle in a microfluidic channel is a combination of optical and drag forces. The optical force can be found by measuring the particle velocity for a certain laser power level and a multiplicative correction factor is applied for the proximity of the particle to the channel surface. This method is not accurate especially for small microfluidic geometries where the particle size is in Mie regime and is comparable to channel cross section. In this work, we propose to use Boundary Element Method (BEM) to simulate fluid flow within the micro-channel with the presence of the particle to predict drag force. Pushing experiments were performed in a dual-beam optical trap and particle's position information was extracted. The drag force acting on the particle was then obtained using BEM and other analytical expressions, and was compared to the calculated optical force. BEM was able to predict the behavior of the optical force due to the inclusion of all the channel walls.
\end{abstract}

Keywords: Microfluidics, dual-beam optical trapping, optical force, boundary element method

\section{INTRODUCTION}

Lab-on-a-chip (LOC) technology and microfluidics enables chemical, biological and physical analyses of bioparticles in miniaturized platforms. Highly selective bio-particle manipulation is necessary for such applications to ensure satisfactory operation. The use of optical forces in microfluidics brings a well-established technology to perform fundamental operations such a trapping, separation, transportation, and sorting in LOC systems. ${ }^{1}$ Optical manipulation could be accomplished via trapping and transporting, deflecting, and pushing particles due to gradient and scattering optical force components. Gradient force created in optical tweezers have been used to trap and transport cells in a microfluidic system, ${ }^{2}$ and separation of a single cell from a population, ${ }^{3}$ while scattering force due to radiation pressure has been utilized for the deflection of cells into a target channel for sorting applications. ${ }^{4}$ Active cell sorting based on both fluorescence sensing ${ }^{5}$ and mechanical properties ${ }^{6}$ have been accomplished using radiation pressure from an expanding optical beam.

Empirical determination of optical force is often needed to calibrate an optical trap and ensure efficient operation of particle/cell manipulation. The external force acting on a trapped particle in fluidic environment is a combination of optical and drag force. Calibration in a petri dish environment can be conducted using Boltzmann statistics or power spectral density function, where position data can be obtained using a quadrant

Further author information: (Send correspondence to M. E. Solmaz)

M. E. Solmaz: E-mail: mehmete.solmaz@ikc.edu.tr, Telephone: +90 (232) 329 3535/3744

B. Çetin: E-mail: barbaros.cetin@bilkent.edu.tr, Telephone: +90 (312) 2902108

B. Baranoğlu: E-mail: besim.baranoglu@atilim.edu.tr, Telephone: +90 (312) 5868680

Optical Trapping and Optical Micromanipulation XII, edited by Kishan Dholakia, Gabriel C. Spalding

Proc. of Vol. 9548, 95481D · C 2015 SPIE · CCC code: 0277-786X/15/\$18 · doi: 10.1117/12.2190319 
photodiode or video microscopy techniques. Both methods aim to fit empirical data to an analytical expression to extract trap stiffness, $k(\mathrm{pN} / \mu \mathrm{m})$, although in a microfluidic environment, the optical trapping force can be over or underestimated based on the method used. ${ }^{7}$ Another method is to measure the escape force velocity of a stationary particle from the optical trap and calculate the viscous drag force. ${ }^{8}$ This calibration method relies on the trapped particle being far away from to the glass surface, $h \gg a$, where $h$ is location of the sphere center from the nearest surface, and $a$ is the particle radius. The drag force acting on a spherical particle in a stagnant fluid can be written as:

$$
\boldsymbol{F}=-\gamma \boldsymbol{u}_{p}
$$

where $\gamma$ is the hydrodynamic drag coefficient and $\boldsymbol{u}_{p}$ is the measured particle velocity. Under the assumption of Stoke's flow, the drag coefficient can be written as:

$$
\gamma=\lambda 6 \pi \eta a,
$$

where $\eta$ is the viscosity and $a$ is the particle radius, and $\lambda$ is a correction factor. In an infinite medium, $\lambda=1.0$, and the equations is knowns as Stoke's law. If the particle surrounded by finite fluid domain, and/or there exists multiple particles, value of $\lambda$ deviates from unity. Some analytical expressions exist for $\lambda$ as a function of diameter-over-separation ratio for applications that include a particle moving parallel or against a single wall. ${ }^{9}$ However, there is no general expression for a particle confined by several walls. For the optical force calibration, an accurate estimation of the drag force on the particle is crucial. For the estimation of drag force, it is a common practice to use Stoke's law ${ }^{10}$ or Faxen formula ${ }^{11}$ in the literature. Strictly speaking, Stoke's law is valid if the particle is moving in an infinite stagnant fluid, and Faxen formula is valid if a particle is flowing parallel to a planar wall in an infinite fluid. Therefore, if the particle size small compared to the micro-channel size, use of Stoke's law is appropriate whereas if the experiments are performed in a petri dish, ${ }^{12}$ Faxen formula is applicable. However, In the case of microfluidic applications, the particles/cells are enclosed in a microchannel at least one dimension of which is comparable to the size of the particle/cell; therefore, the effect of the wall(s) on the drag force needs to be included in the analysis. For an accurate prediction of the drag force on a particle, the hydrodynamic stress tensor on the particle surface needs to be determined and integrated. ${ }^{13}$ In this work, a Boundary Element Method (BEM) based computational model ${ }^{14,15}$ is used to simulate fluid flow within the microchannel with the presence of the particle to predict drag force on a particle. A dual-beam optical trapping setup was fabricated and pushing experiments were performed on a particle with size in Mie regime to extract the position and temporal information. The extracted data was fed into computational model to obtain the drag force on the particle and calibrate the optical trap. The obtained results were also compared to the Stoke's law.

\section{BOUNDARY ELEMENT FORMULATION}

The governing equations for the Stoke's flow is given as ${ }^{15}$

$$
-\nabla P+\mu \nabla^{2} \boldsymbol{u}=0
$$

along with the continuity condition

$$
\nabla \cdot \boldsymbol{u}=0
$$

where $\boldsymbol{u}=\left(u_{1}, u_{2}, u_{3}\right)$ is the velocity field, $\mu$ is the viscosity of the fluid, $P$ is the modified pressure defined in terms of the pressure, $p$, the gravitational acceleration, $\boldsymbol{g}$, the density $\rho$ and the cartesian coordinates $\boldsymbol{x}$ as

$$
P=p-\rho \boldsymbol{g} \cdot \boldsymbol{x}
$$

The boundary element formulation of the Stoke's flow can be written as: ${ }^{15}$

$$
C_{i j}(A) u_{j}(A)=\int_{S} G_{i j}(A, P) t_{j}(P) \mathrm{d} S-\int H_{i j}(A, P) u_{j}(P), \mathrm{d} S
$$

where $A$ represents the fixed (evaluation) point and $P$ represents the varied (integration) point. $C_{i j}$ takes values $1,1 / 2$ or 0 depending on the position of $A$ within the solution domain. ${ }^{15}$ The field variables of Eq. (6) are 
Table 1. Comparison of BEM with Stoke's law

\begin{tabular}{cccc}
\hline Number of Elements & Stoke's law & BEM & \% Error \\
\hline 64 & 18.8496 & 18.3892 & 2.44 \\
128 & 18.8496 & 18.6146 & 1.25 \\
256 & 18.8496 & 18.7304 & 0.63 \\
512 & 18.8496 & 18.7898 & 0.32 \\
1024 & 18.8496 & 18.8197 & 0.16 \\
\hline
\end{tabular}

the velocity components, $u_{i}$, and the traction components, $t_{i}$. In Eq. (6) $G_{i j}$ and $H_{i j}$ are the first and second fundamental solutions of Stoke's equation. Following the discretization of the solution domain, a matrix relation can be obtained as:

$$
\mathbf{H} \cdot \mathbf{u}=\mathbf{G} \cdot \mathbf{t},
$$

where the elements of the matrices $\mathbf{G}$ and $\mathbf{H}$ can be evaluated through the following integral relations:

$$
\begin{aligned}
G_{i j}^{l k} & =\int_{C_{k}} G_{i j}\left(A_{l}, P_{k}\right) \mathrm{d} S, \\
H_{i j}^{l k} & =\int_{C_{k}} H_{i j}\left(A_{l}, P_{k}\right) \mathrm{d} S,
\end{aligned}
$$

where $l$ refers to the element that the fixed point $A$ is on, and $k$ refers to the element being integrated. The matrix relation in Eq. (7) constitutes to a $3 N$ equations of $6 N$ unknowns, $3 N$ of which is to be determined by given boundary conditions. Note that, the imposition of boundary conditions requires the definition of one and only one of the couples $\left(u_{i}^{n}, t_{i}^{n}\right)$ or a combination of these two at all points of the defined boundary, where $n$ represents the node number and $i$ represents the direction. Solving Eq. (7) in view of the imposed boundary conditions, traction components on the boundary of the particle is obtained. The total net drag force on the particle, then, can be obtained through

$$
f_{i}^{B}=\sum_{n=1}^{M} f_{n}=\sum_{n=1}^{M} \int_{C_{n}} t_{i}\left(P_{n}\right) \mathrm{d} S=\sum_{n=1}^{M} t_{i}^{n} A_{n},
$$

where $M$ is the number of nodes on the particle and $A_{n}$ are the areas of the triangular elements on the particle. Note that, in this present study, constant elements are employed to discretize both the channel and the particle boundaries.

\subsection{Code Validation}

To validate the computational model, Stoke's law problem is used as a benchmark problem. A spherical particle with a unit radius is placed in an infinite medium with a free-stream velocity of unity and viscosity of unity. The analytical drag force for this problem can be obtained using Eqs 1 and 2. The comparison of the result of BEM with the analytical solution is given in Table 1. As seen from the Table even with 64 number of elements, BEM-based computational model can predict the drag force with an error of around $2 \%$.

\section{EXPERIMENTAL ANALYSIS}

\subsection{Dual-beam Optical Trap}

First dual-beam optical trap was built by Ashkin in 1970 using CW argon laser and focusing lenses. ${ }^{16}$ The rays hitting the particle surface results in momentum transfer and optical force components pushes the particle to the center of the channel along the optical axis where the radiation pressure from both fibers cancels each other. 


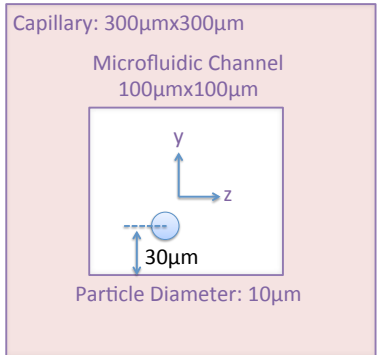

(a)

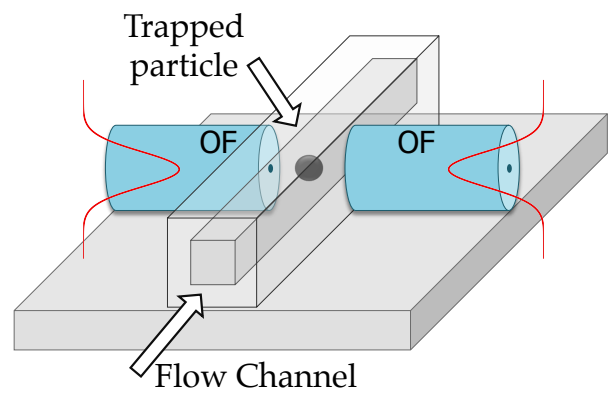

(b)

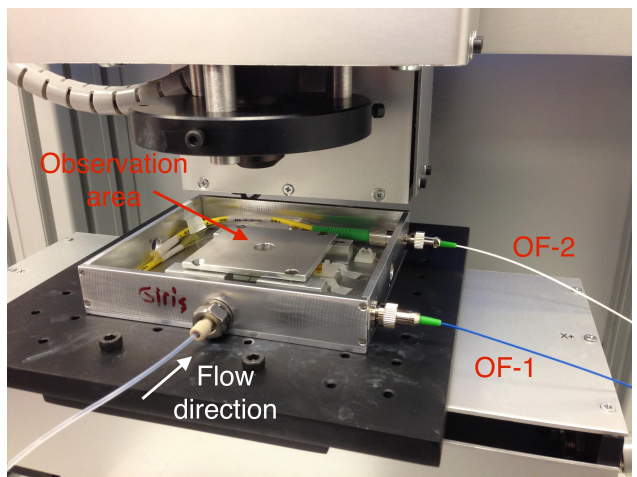

(c)

Figure 1. (a) Schematic drawing of the capillary and microfluidic channel, (b) schematic of the experimental set-up, (c) photograph of the experimental set-up

This is a stable location when there is no fluid flow and lasers are set to the same power level. Although it is possible to build a dual-beam trap using free space optical components, we chose to use optical fibers and took advantage of microfabrication technologies for fiber to channel alignment. In order to trap particles using optical forces, a compact fiber optic based dual-beam optical trap setup was built (Fig. 1). Two single mode fiber coupled semiconductor diode lasers at a wavelength of $807 \mathrm{~nm}$ (Lumics GmbH, Germany) were used as laser sources. The wavelength was chosen to minimize water absorption in the infrared, hence to avoid heating effects. The maximum power out of each fiber was measured to be $150 \mathrm{~mW}$. The fiber ends were coupled to compact DBOT setup and the ends of the bare optical fibers (HI780, Corning, USA) were used as Gaussian light sources for particle trapping. The mode field diameter given by the manufacturers datasheet is $4.8 \pm 0.5 \mu \mathrm{m}$ and the calculated beam diameter at the center of the flow channel is $23.0 \pm 1.0 \mu \mathrm{m}$. The bare ends of the optical fibers were submerged into a index matching gel before placing the next to the flow channel in order to avoid reflections at the air-glass boundary. The lasers were driven by laser controllers (CLD1015, Thorlabs, USA), which were controlled by Labview. A microscope (AIV Labs $\mu$-MIT) was used to observe the experiment, and video was recorded at $90 \mathrm{fps}$ by a CCD camera.

A square capillary channel with $100 \mu \mathrm{m}$ inner diameter and wall thickness (Fig. 1-(a)) was placed perpendicular to the optical fibers on the micro-fabricated alignment chip (Fig. 1-(b)) which was placed under the metal plate. The capillary was used as a flow channel to transport particles to the trapping site by adapting to the custom-made DBOT housing using steel connectors which was placed under a customized microscope (Fig. 1-(c)) and was connected to a peristaltic pump (Ismatech, USA) using microfluidic adapters. A shutoff valve was used between the pump and the capillary channel to stop the flow when needed. Fiber optical connectors were used to couple both lasers to the custom DBOT setup.

\subsection{Pushing Experiments}

To calculate the drag force acting upon the trapped particle, we performed pushing experiments ${ }^{11}$ on polystyrene particles with a diameter of $\sim 10 \mu \mathrm{m}$. The refractive index of the particle was given as 1.61 by the manufacturer. The fluid flow was stopped when the particle was near the trapping region. The optical power was set to $20 \mathrm{~mW}$ and trapping was achieved at the center of the channel. By increasing the power of only one laser to $50-100 \mathrm{~mW}$, the particle was pushed to other side of the channel, almost touching the channel wall. The same laser was turned off suddenly by software and the particle was pushed to the other wall by the optical radiation pressure while temporal information was recorded. The pushing experiments were repeated with 50 and $100 \mathrm{~mW}$ power coming out of the fibers. The recorded videos were then processed frame by frame by ImageJ software to extract the particle location with respect to walls. The velocity data was found to calculate the drag force. The viscosity of the water was taken $0.001 \mathrm{~Pa} \cdot \mathrm{s}$. 

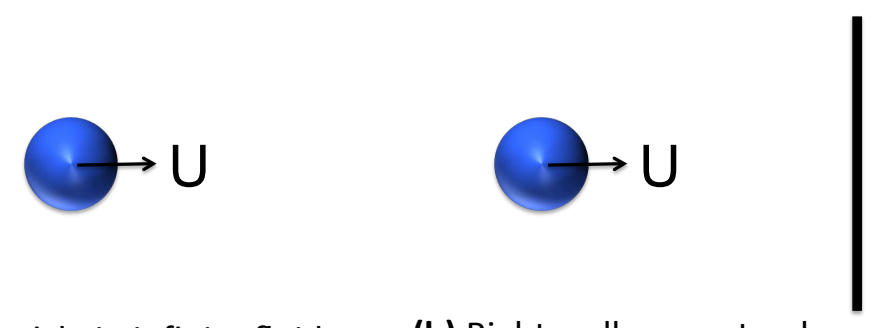

(a) Particle in infinite fluid

(b) Right wall present only
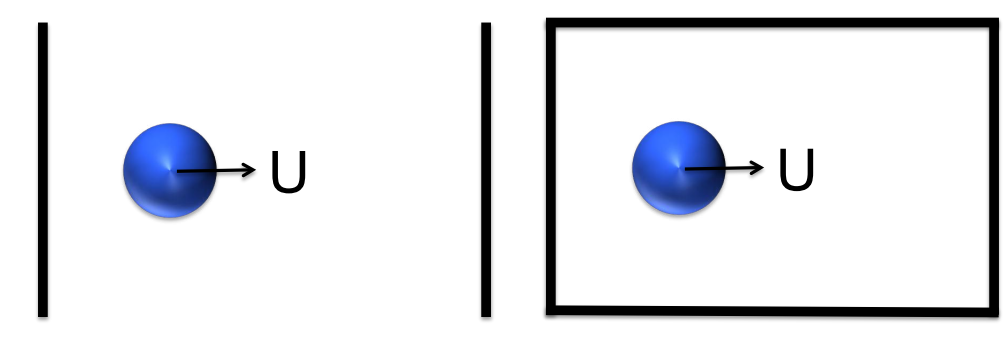

\section{(c) Right \& left wall present only (d) Particle in micro-channel}

Figure 2. Schematics of the different cases used in the simulations

\subsection{Data Processing and Force Calculation}

Once the position vs time data was generated, a fourth-order polynomial was fitted on the data, and the velocity values were obtained from the derivative of the polynomial at the measurement points. Ignoring the inertia of the particle and assuming the velocity data corresponds to the terminal speed of the particle at the given location, the drag force on the particle was determined using boundary element code by assigning the velocity data on the particle boundary. This analysis was performed for several different cases: (a) particle is infinite fluid, (b) particle is flowing against a single wall (right wall) in z-direction, (c) particle is flowing against the right wall with the presence of left wall (fluid is infinite in y-direction), (d) particle is flowing against the right wall in a micro-channel. Note that if a wall is absent fluid domain is infinite in that direction. The schematics of these cases are shown in Fig. 2. 306 elements were used to discretize the sphere (Case-a). 952 elements for the particle with right wall only (Case-b), 1296 elements for the particle with right and left wall (Case-c), and 1984 elements for the particle in micro-channel (Case-d) were used. Simulation were performed on MacBoook Pro desktop computer $(2.3 \mathrm{GHz}$ Intel Core i7, $16 \mathrm{~GB} 1600 \mathrm{MHz}$ DDR3). The simulations with the largest number of elements took approximately 5 mins for a given particle location. A picture of the mesh used in the simulations can be seen in Fig. 3.

For comparison, drag force was also calculated based on Faxen formula, which gives an analytical expression for the drag force on the sphere flowing parallel to a planar wall, and Brenner formula, which gives an analytical expression for the drag force on the sphere flowing against a planar wall. The correction factor for Faxen formula can be written as, ${ }^{9}$

$$
\lambda=\frac{1}{1-\frac{9}{16}\left(\frac{a}{h}\right)+\frac{1}{8}\left(\frac{a}{h}\right)^{3}-\frac{45}{256}\left(\frac{a}{h}\right)^{4}-\frac{1}{16}\left(\frac{a}{h}\right)^{5}}
$$

while the correction factor for Brenner formula can be written as: ${ }^{9}$

$$
\lambda=\frac{4}{3} \sinh \alpha \sum_{n=1}^{\infty} \frac{n(n+1)}{(2 n-1)(2 n+3)}\left[\frac{2 \sinh (2 n+1) \text { alpha }+(2 n+1) \sinh 2 \alpha}{4 \sinh ^{2}\left(n+\frac{1}{2}\right) \alpha-(2 n+1)^{2} \sinh ^{2} \alpha}-1\right]
$$

To compare the drag force results with the estimated optical force, we also calculated the scattering optical force on the particle by using ray optics ${ }^{11,17}$ by assuming the particle was placed along the optical axis. The 


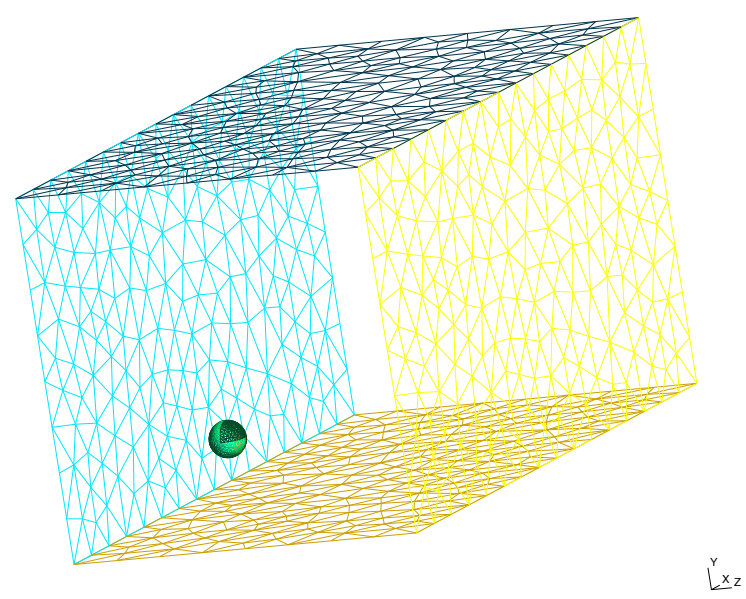

Figure 3. Mesh used in the simulations

particle was treated as a spheroid with equal major and minor axes. The rays entering the particle propagated through 5 internal reflections inside the particle, each of which causes a loss in momentum. The effective force on unit area was calculated and was repeated for the front and back surfaces to obtain the total force from one laser beam.

\section{RESULTS AND DISCUSSION}

The position data for $50 \mathrm{~mW}$ and $100 \mathrm{~mW}$ can be seen in Fig. 4-(a) and Fig. 5-(a), respectively. As seen form the figure, $4^{t h}$-order polynomial fits the data quite well (with and $R^{2} \sim 0.999$ ). Results for drag force calculations (BEM, Faxen formula and Brenner formula) together with the optical force is illustrated in Fig. 4-(b) and Fig. 5(b) for $50 \mathrm{~mW}$ and $100 \mathrm{~mW}$, respectively. For the BEM simulations, aforementioned four cases were used. Several observations can be deduced from the figures:

- The important and expected observation is the increase of drag force with the inclusion of the walls for $50 \mathrm{~mW}$ and $100 \mathrm{~mW}$ cases due to the hydrodynamic interaction of the particle with the walls.

- Optical force monotonically decreases as the particle moves far away from the laser source due to a decrease in optical power and Gaussian beam expansion. Stoke's law was not able to predict the optical force behavior, instead it predicts a plateau followed by a decrease. Faxen formula introduces a correction as a function of spacing between the particle and the bottom wall; therefore it predicts the same behavior with the Stoke's law, and was also not able to predict the same behavior as the optical force .

- BEM simulations with right channel wall only (Case-b, red curve) was able to give a similar result with the Brenner formula (black curve), although the behavior of the optical force is not achieved. Both results predict an increase in the drag force as the particle is in the close vicinity of the right wall due to the hydrodynamic interaction of the particle with the wall. Similarly, the same increase is also observed for the cases with the left wall included (Case-c, blue curve) and all walls included (Case-d, green curve). This behavior is not expected since the optical force tend to decrease with distance. One possible cause of this inconsistency is due to the neglecting the rotation of the particles in our analysis. The rotation of the particle could not be observed during our experiments due to fact that the polystyrene particles used were spherical.

- With the inclusion of the left wall, the monotonic decrease of the optical force was observed. The inclusion of the top and bottom walls further increases the drag force. At the both ends the blue curve and green curve approaches to the same value, which is quite expected since the the effect of the side walls have a stronger effect on the drag force compared to that of the upper and the lower wall. 


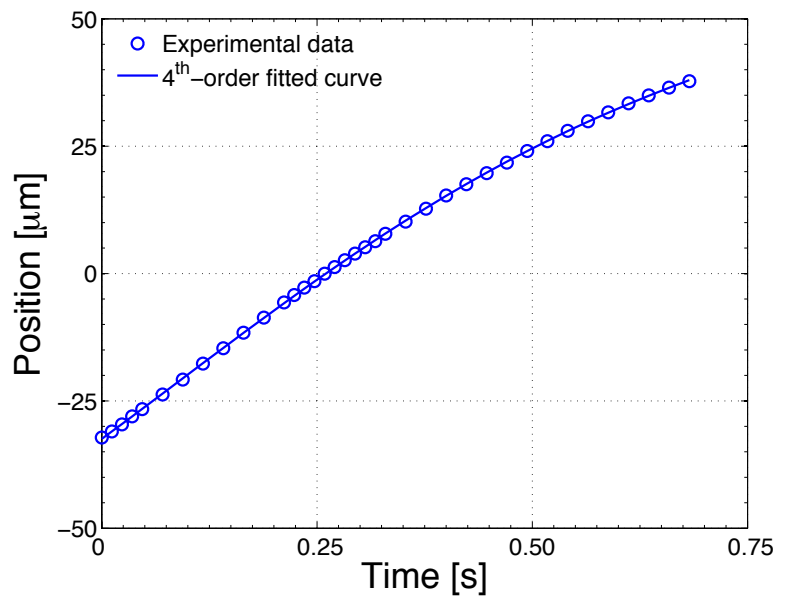

(a)

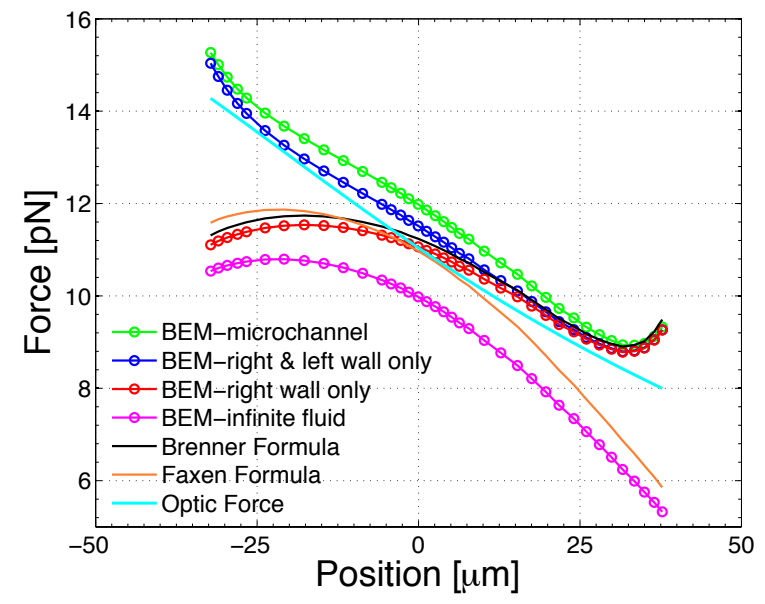

(b)

Figure 4. Results for $50 \mathrm{~mW}$ experiments: (a) Position, (b) Force data

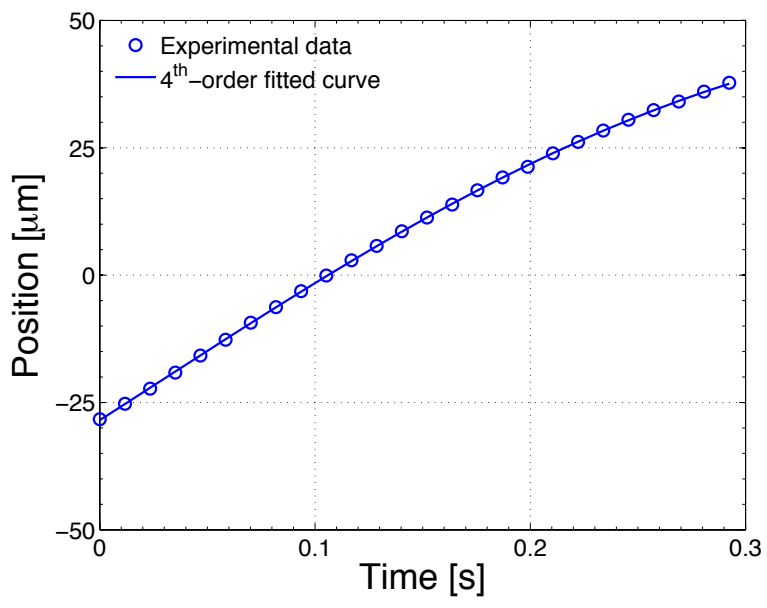

(a)

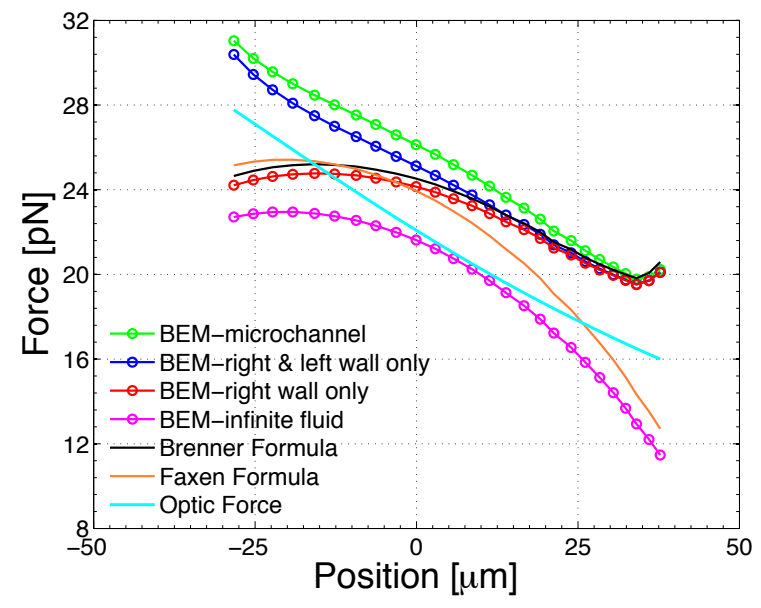

(b)

Figure 5. Results for $100 \mathrm{~mW}$ experiments: (a) Position, (b) Force data

- The most important conclusion of these figures is that the use of analytical expressions for the optical force calibration is not appropriate for the particles trapped/manipulated in micro-channels. The effect of the hydrodynamic interaction of the particle with the walls needs to be included in the analysis which can only be performed by some numerical techniques. BEM is a convenient option for these kinds of applications considering the linear nature of the governing equations. Moreover, the well-known analytical expressions (i.e. Faxen and Brenner formulas) for the correction factor do not take into account the rotation of the particle. There is a high possibility that the due to the non-symmetric flow field (mainly due the presence of the walls), the particle would rotate and that would change the drag force on the particle. In a future study, we would like to include the rotation by modeling the particle as a freely rotating particle.

\section{ACKNOWLEDGMENTS}

Dual-beam optical trap device development (M. E. S.) was funded TUBITAK 2232 program (113C001) and TUBITAK 113E321 project. Dr. Solmaz would like to thank Dr. Michelle Povinelli and Roshni Biswas of University of Southern California for their assistance in optical force calculations. 


\section{REFERENCES}

[1] Cetin, B., Ozer, M. B., and Solmaz, M. E., "Microfluidic bio-particle manipulation for biotechnology," Biochem. Eng. J. 92, 63-82 (2014).

[2] Eriksson, E., Enger, J., Nordlander, B., Erjavec, N., Ramser, K., Goksr, M., Hohmann, S., Nystrm, T., and Hanstorp, D., "A microfluidic system in combination with optical tweezers for analyzing rapid and reversible cytological alterations in single cells upon environmental changes," Lab Chip 7(1), 71-76 (2006).

[3] Hellmich, W., Pelargus, C., Leffhalm, K., Ros, A., and Anselmetti, D., "Single cell manipulation, analytics, and label-free protein detection in microfluidic devices for systems nanobiology," Electrophoresis 26(19), 3689-3696 (2005).

[4] Wang, M. M., Tu, E., Raymond, D. E., Yang, J. M., Zhang, H., Hagen, N., Dees, B., Mercer, E. M., Forster, A. H., Kariv, I., Marchand, P. J., and Butler, W. F., "Microfluidic sorting of mammalian cells by optical force switching," Nat Biotech 23(1), 83-87 (2005). 10.1038/nbt1050.

[5] Bragheri, F., Minzioni, P., Martinez Vazquez, R., Bellini, N., Paie, P., Mondello, C., Ramponi, R., Cristiani, I., and Osellame, R., "Optofluidic integrated cell sorter fabricated by femtosecond lasers," Lab on a Chip 12(19), 3779-3784 (2012).

[6] Bragheri, F., Pai, P., Yang, T., Nava, G., Vzquez, R. M., Di Tano, M., Veglione, M., Minzioni, P., Mondello, C., and Cristiani, I., "Sorting on the basis of deformability of single cells in a femtosecond laser fabricated optofluidic device," in [SPIE LASE], 93550G-93550G-6, International Society for Optics and Photonics (2015).

[7] Uhrig, K., Kurre, R., Schmitz, C., Curtis, J. E., Haraszti, T., Clemen, A. E. M., and Spatz, J. P., "Optical force sensor array in a microfluidic device based on holographic optical tweezers," Lab on a Chip $\mathbf{9}(5)$, 661-668 (2009).

[8] Svoboda, K. and Block, S. M., "Biological applications of optical forces," Annual Review of Biophysics and Biomolecular Structure 23(1), 247-285 (1994).

[9] Happel, J. and Brenner, H., [Low Reynolds number hydrodynamics: with special applications to particulate media], vol. 1, Springer Science \& Business Media (1983).

[10] Lincoln, B., Schinkinger, S., Travis, K., Wottawah, F., Ebert, S., Sauer, F., and Guck, J., "Reconfigurable microfluidic integration of a dual-beam laser trap with biomedical applications," Biomedical Microdevices $\mathbf{9}(5)$, 703-710 (2007).

[11] Solmaz, M. E., Sankhagowit, S., Biswas, R., Mejia, C. A., Povinelli, M. L., and Malmstadt, N., "Optical stretching as a tool to investigate the mechanical properties of lipid bilayers," RSC Advances 3(37), 1663216638 (2013).

[12] Guck, J., Ananthakrishnan, R., Mahmood, H., Moon, T. J., Cunningham, C. C., and Kas, J., "The optical stretcher: a novel laser tool to micromanipulate cells," Biophysical Journal 81(2), 767-784 (2001).

[13] Cetin, B. and Li, D., "Dielectrophoresis in microfluidics technology," Electrophoresis 32, 2410-2427 (2011).

[14] Baranoglu, B. and Cetin, B., "A particle flow specific boundary element formulation for microfluidic applications," in [4th Micro and Nano Flow Conference, 6-10 September 2014], Karayiannis, T., Konig, C. S., and Balabani, S., eds., (206), Brunel University (2014).

[15] Karakaya, Z., Baranoglu, B., Cetin, B., and Yazici, A., "Microfluidic bio-particle manipulation for biotechnology," CMES: Computer Modeling in Engineering \& Sciences 104(3), 227-249 (2015).

[16] Ashkin, A., "Acceleration and trapping of particles by radiation pressure," Physical Review Letters 24(4), 156 (1970).

[17] Sosa-Martinez, H. and Gutierrez-Vega, J., "Optical forces on a spheroidal microparticle using a classical optics approximation," in [Proc. SPIE], 7400(1), 74002B, International Society for Optics and Photonics (2009). 